Ann. Biol. anim. Bioch. Biophys., I964. 4 (3), 239-244.

\title{
ACTIVITÉ PROTÉOLYTIQUE DE L'APPAREIL GASTRIQUE D'OISEAUX GRANIVORES ET CARNIVORES
}

\author{
Claire HERPOL \\ Laboraloire de Zoophysiologie de l'Université de Gand (Belgique) \\ 14, rue de l'Université, Gand (Belgique) \\ Directeur : $P^{\mathrm{r}} D^{\mathrm{r}} G$. Van Grembergen
}

SOMMAIRE

Les données quantitatives sur le contenu enzymatique des estomacs des oiseaux que l'on trouve dans la littérature sont généralement peu précises et de plus, concernent principalement les granivores.

L'activité protéolytique d'extraits de tissus du ventricule succenturié et du gésier d'espèces granivores et carnivores a été déterminée par la méthode de titration au formol selon SöRENSEN.

Le ventricule succenturié est le siège d'une haute activité pepsique, plus encore chez les oiseaux carnivores que chez les granivores.

Le gésier des granivores ne contient pas d'enzymes protéolytiques. Dans celui des oiseaux carnivores, par contre, nous avons trouvé une activité protéolytique certaine, que la littérature spécialisée ne mentionne nulle part.

\section{INTRODUCTION}

L'appareil gastrique des oiseaux se compose de deux parties distinctes, tant sur le plan morphologique que sur le plan physiologique. Ce sont le ventricule succenturié ou estomac glandulaire et l'estomac musculaire ou gésier.

Sur le plan physiologique il est généralement reconnu que le ventricule succenturié a pour fonction unique de former les composants du suc gastrique, puisque la nourriture ne séjourne que quelques instants dans ce compartiment (STEINMETZER, I924). Le gésier par contre ne participe nullement à l'élaboration d'enzymes mais il est le siège de la digestion par la pepsine, principal composant du suc gastrique, qui y trouve des conditions d'acidité favorables (HERPOL et VAN GREMBERGEN, I96r). Les glandes du gésier contribuent uniquement à la formation d'un revê- 
tement intérieur de koïline. La présence de ce revêtement et l'action de la masse musculaire du gésier aident à mener à bien la fonction mécanique de cet organe, laquelle est particulièrement importante chez les granivores.

L'appareil gastrique des Rapaces se présente d'une façon un peu différente, surtout sur le plan morphologique. Le ventricule succenturié forme le suc gastrique et celui-ci exerce son action dans le gésier, mais aussi dans la cavité même du ventricule succenturié, les proies des Rapaces étant souvent trop grandes pour être contenues par le gésier, même s'il est extensible. La masse musculaire du gésier est en effet fort peu développée et l'organe broyeur caractéristique des granivores se présente ici comme une simple poche dilatable dont le revêtement intérieur de koïline est peu important ou même pratiquement inexistant.

La littérature ne nous offre que fort peu de données quantitatives sur le contenu enzymatique des organes digestifs des oiseaux ; ces données concernent principalement les granivores.

Le but du présent travail a été l'étude comparative et quantitative de l'activité protéolytique des estomacs d'espèces granivores et carnivores (Rapaces).

\section{MATÉRIEL, ET TECHNIQUES}

Les recherches ont été effectuées chez cinq espèces d'oiseaux, dont deux granivores, le Poulet (Gallus domesticus) et le Pigeon domestique (Columba livia domestica) et trois Rapaces, dont deux diurnes, le Faucon crécelle (Falco tinnunculus tinnunculus L.), la Buse variable (Buteo buteo buteo L.) et un Rapace nocturne, la Chouette chevêche (Athene noctua vidalii BREHM). Pendant la courte période passée en laboratoire les oiseaux furent nourris respectivement de graines commerciales et de viande rouge.

L'oiseau est tué par décapitation, après un jeûne de 24 heures, ce qui nous permet d'estimer le contenu enzymatique des glandes stomacales dans des conditions comparables. Le ventricule succenturié et le gésier sont prélevés séparément, soigneusement lavés, puis séchés sur du papier filtre. Après détermination du poids frais, ils sont homogénéisés avec I $30 \mathrm{ml}$ de solution $\mathrm{NaCl}_{7} \mathrm{P}$. I ooo dans un appareil Ultra-turrax. L'extrait ainsi obtenu est employé comme tel ou conservé en glacière pendant au maximum $3^{6}$ heures en y ajoutant un peu de thymol prour prévenir la décomposition.

L'activité protéolytique des extraits a été déterminée par la méthode de titration au formol selon SöRENSEN. Nous avons suivi la prescription de RONA (1931, p. 259 et 289) mélangeant $50 \mathrm{ml}$ de solution de caséine (d'après HaMmarsten de la firme Merck $\mathrm{n}^{\circ}$ 2242) et $10 \mathrm{ml}$ d'extrait de tissu stomacal. Le $\mathrm{pH}$ est ajusté à l'aide de $\mathrm{NaOH} \mathrm{N}$ ou de $\mathrm{HCl} \mathrm{N}$ aux valeurs désirées, allant de $\mathrm{pH}$ i, o à $\mathrm{pH} 9,0$. Les mesures de $\mathrm{pH}$ ont été effectuées avec un $\mathrm{pH}$-mètre Beckman modèle $\mathrm{G}$ équipé de ses électrodes classiques. Au début de l'expérience et après 24 heures d'incubation à $40^{\circ} \mathrm{C}$, correspondant à la température corporelle élevée des oiseaux, on prélève $20 \mathrm{ml}$ du mélange précité et l'on y ajoute $10 \mathrm{ml}$ d'une solution de formol contenant de la phénolphtaléine. La titration se fait alors avec une solution de $\mathrm{NaOH}$ o, $2 \mathrm{~N}$. La différence entre les deux titrations donne l'activité protéolytique de l'extrait. Chaque essai est accompagné d'un contrôle pour éviter les erreurs dues à l'autodigestion de la caséine, l'extrait étant remplacé par ıo $\mathrm{ml}$ d'eau distillée.

\section{RÉSULTATS}

Les résultats obtenus sont rassemblés dans les tableaux $x$ et 2 . Les valeurs du $\mathrm{pH}$ sont celles mesurées au début de l'expérience, car nous avons pu constater qu'elles restent relativement constantes, ce qui fait que nous avons estimé inutile l'addition de solutions tampons, qui pourraient par ailleurs présenter le danger 
d'influencer la protéolyse. L'activité protéolytique de l'extrait stomacal est exprimée en $\mathrm{ml}$ de solution $\mathrm{NaOH} 0,2 \mathrm{~N}$, calculés par gramme de poids frais, pour permettre une comparaison aisée.

\section{TABLEAU I}

Activité des enzymes protéolytiques du ientricule succenturié d'espèces granivores et carnivores (Rapaces) En ml. $\mathrm{NaOH} 0,2 \mathrm{~N}$ par $\mathrm{g}$. de poids frais.

\begin{tabular}{|c|c|c|c|c|c|c|c|c|c|}
\hline $\mathrm{pH} \rightarrow$ & 1 & 2 & 3 & 4 & 5 & 6 & 7 & 8 & 9 \\
\hline $\begin{array}{l}\text { Gallus domesticus (moyenne de } \\
10 \text { indiv.) } \ldots \ldots \ldots \ldots \ldots \ldots\end{array}$ & 62.9 & 61,3 & 57,5 & 59,6 & 51,0 & 15,1 & 3,7 & 3,5 & 0,7 \\
\hline $\begin{array}{l}\text { Columba l. domestica (moyenne } \\
\text { de } 10 \text { indiv.) ............ }\end{array}$ & 292,7 & 213,8 & 138,3 & 49,1 & 14,2 & 19,3 & 20,8 & 9,8 & 0,6 \\
\hline 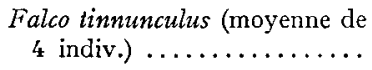 & 418,8 & 320,4 & 237,3 & 127,8 & 91,7 & 23,3 & 28,7 & 25,3 & 8,3 \\
\hline Buteo buteo ( 1 individu) ..... & 117,9 & 108,2 & 93,9 & 66,9 & 69,4 & 12,7 & 18,4 & 4,9 & 0,4 \\
\hline Athene noctua ( 1 individu) ... & 373,1 & 88,5 & 150,0 & 150,0 & 69,2 & 15,4 & 3,8 & 15,4 & 3,8 \\
\hline
\end{tabular}

TABLEAU 2

Activité des enzymes protéolytiques du gésier d'espèces granivores et carnivores (Rapaces) En ml. NaOH $0,2 \mathrm{~N}$ par g. de poids frais.

\begin{tabular}{|c|c|c|c|c|c|c|c|c|c|}
\hline $\begin{array}{cc}\text { Espèces } & \mathrm{pH} \rightarrow \\
\downarrow & \\
\end{array}$ & 1 & 2 & 3 & 4 & 5 & 6 & 7 & 8 & 9 \\
\hline Gallus domesticus ............ & $\begin{array}{l}0,3 \\
0 \\
1,2 \\
0 \\
0\end{array}$ & $\begin{array}{l}2,1 \\
0,3 \\
0 \\
0 \\
0,2\end{array}$ & $\begin{array}{l}1,8 \\
0,9 \\
0,6 \\
0,2 \\
0,4\end{array}$ & $\begin{array}{l}6,6 \\
1,8 \\
1,3 \\
0,5 \\
1,5\end{array}$ & $\begin{array}{l}6,8 \\
0 \\
1,1 \\
1,0 \\
1,3\end{array}$ & $\begin{array}{l}5,4 \\
4,3 \\
0 \\
0,2 \\
0\end{array}$ & $\begin{array}{l}6,3 \\
4,3 \\
2,9 \\
0,6 \\
0,6\end{array}$ & $\begin{array}{l}0,8 \\
5,5 \\
1,8 \\
0,3 \\
0,4\end{array}$ & $\begin{array}{l}0,8 \\
0,6 \\
1,7 \\
0 \\
0\end{array}$ \\
\hline Columba domestica ............ & $\begin{array}{c}0 \\
5,2 \\
0 \\
19,4 \\
14,4\end{array}$ & $\begin{array}{l}0,9 \\
0,8 \\
0 \\
4,1 \\
5,4\end{array}$ & $\begin{array}{l}0,7 \\
0,7 \\
6,4 \\
4,9 \\
6,4\end{array}$ & $\begin{array}{l}1,9 \\
1,1 \\
1,7 \\
3,7 \\
4,2\end{array}$ & $\begin{array}{l}0 \\
2,8 \\
0 \\
6,0 \\
3,2\end{array}$ & $\begin{array}{l}1,4 \\
4,8 \\
8,1 \\
7,1 \\
6,4\end{array}$ & $\begin{array}{r}10,8 \\
10,9 \\
5,1 \\
8,6 \\
2,9\end{array}$ & $\begin{array}{l}0,2 \\
3,7 \\
3,8 \\
4,8 \\
3,8\end{array}$ & $\begin{array}{l}0,5 \\
1,1 \\
0 \\
0 \\
1,0\end{array}$ \\
\hline Falco tinnunculus ........... & $\begin{array}{l}110,0 \\
166,7 \\
125,5 \\
112,2\end{array}$ & $\begin{array}{r}124,7 \\
126,1 \\
63,3 \\
104,0\end{array}$ & $\begin{array}{l}73,7 \\
36,4 \\
38,9 \\
43,2\end{array}$ & $\begin{array}{l}26,8 \\
64,2 \\
25,5 \\
70,3\end{array}$ & $\begin{array}{r}29,5 \\
33,9 \\
16,7 \\
6,7\end{array}$ & $\begin{array}{r}19,5 \\
17,0 \\
12,2 \\
4,1\end{array}$ & $\begin{array}{r}1,6 \\
24,2 \\
7,8 \\
6,7\end{array}$ & $\begin{array}{r}3,1 \\
24,2 \\
20,0 \\
4,0\end{array}$ & $\begin{array}{c}1,0 \\
10,9 \\
0 \\
8,1\end{array}$ \\
\hline Buteo buteo............ & 92,3 & 54,7 & 31,2 & 13,6 & 15,5 & 15,7 & 18,9 & 2,4 & 1,9 \\
\hline Athene noctua ............. & 50,7 & 22,5 & 3,5 & 3,5 & 2,1 & 0 & 2,8 & 1,4 & 2,1 \\
\hline
\end{tabular}




\section{DISCUSSION}

Il ressort des tableaux I ct 2 qu'il existe une activité protéolytique plus ou moins élevée en milieu acide. La majeure partie de cette activité est sans aucun doute due à l'action de pepsines. Il est néanmoins certain que d'autres enzymes, présents dans les extraits de tissus, sont en partie responsables de l'action caséinolytique. Ainsi, 1'hydrolyse dans la région allant de $\mathrm{pH} 4,0$ à $\mathrm{pH} 5,0$ est partiellement due à la présence de cathepsines, tandis que d'éventuelles exopeptidases peuvent également contribuer à la caséinolyse, dans une mesure dépendante des conditions de pH. Selon DE RyckF (Ig62) l'activité optimale de la dipeptidase gastrique du poulet (substrat : glycyl-glycine) se situe aux environs de $\mathrm{pH} 8,5$. Le même auteur détermina les activités d'amino- et carboxypeptidases respectivement aux valeurs de $\mathrm{pH} 8$,o et 4,2 .

Ces diverses considérations nous amènent à conclure que l'activité protéolytique notée aux $\mathrm{pH}$ les plus acides ( $\mathrm{pH}$ I et 2 ) ne peut pratiquement être due qu'à une activité pepsique. En effet selon les données de la littérature le pH-optimum se situe aux environs de valeurs I,5 à 2,2 .

Que le ventricule succenturié soit une source particulièrement riche en protéinase active en milieu acide n'a jamais été controversé et nos résultats dans ce domaine sont en parfaite concordance avec les đonnées de la littérature (STURKIE, I954-FARNER, I960, etc.). Cependant lorsqu'il s'agit de résultats quantitatifs nous nous trouvons la plupart du temps devant des données peu précises. Plimmer et ROSEDALE (I922) font état de digestion plus ou moins rapide, tandis que HEWITT et SchELKOPF (I955) donnent une vague idée quantitative en discernant une activité petite, moyenne et grande, tout en se limitant à l'étude comparative des divers organes du tractus digestif de poulets seulement. Il en est de même pour VERHEYEN (I943) qui constate que le suc gastrique des Rapaces diurnes est plus puissant que celui des Hiboux.

FrIEDMan (I939), par contre, compare les teneurs en pepsine de sucs gastriques, sécrétés à la suite d'injections combinées d'acétylcholine et d'histamine, et constate une concentration plus élevée ( $\mathrm{I} 024$ unités Mett) chez le Poulet, que chez le Pigeon (I44 unités Mett); R. JeUniaux (I959) fait cepenđant remarquer que si l'on tient compte de la teneur relative par rapport all poids de l'organe, la quantité de pepsine est plus grande chez le Pigeon.

Pour les deux granivores étudiés, nous ne pouvons que confirmer les résultats de R. Jeuniaux. La comparaison des moyennes du tableau I les concernant montre en outre que l'activité peut varier đu simple au quintuple, avec une réserve résultant d'une part de certaines variations individuelles reconnues dans ce domaine et d'autre part du degré d'imprécision de la méthode. Remarquons cependant que nos résultats montrent une réelle concordance au sein d'une même espèce.

Les Rapaces semblent avoir une teneur particulièrement haute en pepsine. Nous voulons limiter notre comparaison au Faucon. Si nous avons communiqué les résultats obtenus sur deux autres espèces, c'est simplement à titre d'information, puisqu'il serait dangereux de baser une conclusion sur les résultats d'une seule expérience. La 
haute teneur en protéinases est probablement à considérer comme une corrélation entre 1'équipement enzymatique et le régime alimentaire carnivore, riche en protéines. C. JEuniaux (I96I) a remarqué que si les corrélations entre la morphologie de 1'appareil digestif et le régime alimentaire sont évidentes, l'adaptation des sécrétions digestives des vertébrés à leur régime alimentaire, se limite à certaines variations quantitatives, dont il n'est pas aisé de préciser l'importance.

Le résultat le plus surprenant de nos expériences est d'avoir mis en relief l'activité protéolytique certaine de l'estomac, dit musculaire, des Rapaces. Rappelons que de nombreux chercheurs ont mis l'accent sur l'absence d'enzymes dans la muqueuse du gésier du Poulet et ont prouvé que Yamamota (cf. von BudDenBRock I956, p. 388) avait tort quand il écrivait en I937 que le gésier du Poulet sécrétait de la pepsine. Nos résultats sur les espèces granivores confirment d'ailleurs leur thèse. Mais il serait dangereux de généraliser ces conclusions en las appliquant aux espèces carnivores, tel que le fait Browne (r922), p. exemple, qui estime que le gésier des oiseaux carnivores ne doit être considéré que comme une expansion membraneuse du ventricule succenturié, libre en tout cas de toute muqueuse sécrétoire.

Le tableau 2 montre clairement que nous nous trouvons devant une activité protéolytique nette chez les oiseaux rapaces et prouve en outre que ces résultats sont individuellement reproductibles. I a protéinase à optimum acide formée dans le gésier ne peut certes qu'améliorer le rendement de la digestion des proies de ces oiseaux.

Rę̧u pour publication en airril 1964

\section{SUMMARY}

PROTEOLYTIC ACTIVITY IN THE STOMACH OF GRANIVOROUS AND CARNIVOROUS BIRDS

The literature is very poor in quantitative values of proteolytic activity of the digestive tract of birds, and furthermore most of the available data concern granivorous birds.

Using SöRENSEN's formoltitration, we have attempted to determine the proteolytic activity of the glandular and the muscular stomach of granivorous and carnivorous birds.

The results expressed in cc $\mathrm{NaOH} 0,2 \mathrm{~N} / \mathrm{g}$ fresh tissue are given in tables $\mathrm{I}$ and 2.

As expected, the glandular stomach shows a high proteolytic activity in acid medium. Carnivorous birds have a higher pepsin content than granivorous, which probably is to be brought in correlation with the high protein content of the food. The glandular stomach of the pigeon contains about 5 times as much enzyme as that of the chicken (table r).

The muscular stomach of granivorous birds does not contain any enzyme. On the contrary that of carnivorous species has a real pepsine action (table 2). This was the most surprising result of our experiences, for it was never stated in the literature concerning digestion of birds.

\section{RÉFÉRENCES BIBLIOGRAPHIOUES}

Browne T. G., igz2. Some observations on the digestive system of the fowl. J. Comp. Path. Ther., 35, I 2-32.

Buddenbrock W. von, 1956. Vergleichende Physiologie, vol. III, p. 388, Birkhauser Verlag. Basel und Stuttgart.

De Rycke P., Ig62. Onderzoek over exopeptidasen bij het kuiken. Natuuraet. Tijdschr., 43, 82-86. 
C. HERPOI,

Farner D. S., 1960. In Marshall A. J. Biology and comparative physiology of birds, vol, I, 4 r I-449 Academic Press, New York.

Friedman M. H. F., 1939. Gastric secretion in birds. J. Cell. Comp. Physiol., 13, 219-234.

Herpol C., Van Grembergen G., 196r. Le pH dans le tube digestif des Oiseaux. Ann. Biol. anim. Bioch. Biophys., 1, 31 7-321.

HewiTT E. H., SCHELKOPF L., I955. pH values and enzymatic activity of the digestive tract of the chicken. Am. J. Vet. Res., 16, $576-579$.

Jeuniaux C., ig6r. Digestion de la chitine chez les Oiseaux et les Mammifères. Ann. Soc. Roy. Zool. Belg., 92, 27-45.

JEUniauX R., r959. Bijdrage tot de kennis der eiwitsplitsende fermenten bij vogels. Thesis Rijksuniversiteit Gent.

Plimmer R. H. A., Rosedale J. L., I922. Distribution of enzymes in the alimentary canal of the chicken. Bioch. J., 16, 23-26.

Rona P., I931. Praktikum der Physiologischen Chemie, vol. I, p. 259 et 289. Springer Verlag, Berlin.

Steinmetzer K., 1924. Die zeitlichen Verhältnisse beim Durchwandern von Futter durch den MagenDarmkanal des Huhnes. Pfiuger's Arch. Ges. Physiol., 206, 500-505.

Sturkie P. D., 1954. Avian Physiology. Comstock Publ. Assoc., Ithaca, New York.

Verheyen R., I943. De dag-en nachtroofvogels van België. Uilg. door Kon. Natuurhist. Mus. van België. russel. 